\title{
Chitinase-3-like Protein 1 (YKL-40) Is Expressed in Lesional Skin in Hidradenitis Suppurativa
}

\author{
JOANNA SALOMON $^{1}$, ALEKSANDRA PIOTROWSKA $^{2}$, ŁUKASZ MATUSIAK $^{1}$, \\ PIOTR DZIĘGIEL ${ }^{2}$ and JACEK C. SZEPIETOWSKI ${ }^{1}$ \\ ${ }^{1}$ Department of Dermatology, Venereology and Allergology, Wrocław Medical University, Wrocław, Poland; \\ ${ }^{2}$ Department of Human Morphology and Embryology, Wrocław Medical University, Wroctaw, Poland
}

\begin{abstract}
Background/Aim: Hidradenitis suppurativa (HS) is a chronic disease. Recently, we suggested, chitinase-3-like protein 1 (YKL-40) as a new biomarker for HS since its serum levels were found to be elevated in patients. The objective of this study was to examine YKL-40 tissue expression in HS. Materials and Methods: The study was conducted on 7 patients with HS. The biopsies were obtained from lesional and non-lesional skin. The tissue expression of $Y K L-40$ was assessed using an immunohistochemical method. Results: Inflammatory infiltrates of various grades were visible in all samples of lesional skin. The inflammatory cells within those infiltrates showed a strong cytoplasmic expression of YKL-40. In non-lesional HS skin the pattern of $Y K L-40$ expression was similar to that observed in healthy skin. Conclusion: Inflammatory infiltrates in HS skin lesions may be responsible for the elevated YKL-40 serum level. $Y K L-40$ can be considered one of the most valuable biomarkers of $H S$.
\end{abstract}

Hidradenitis suppurativa (HS) is a chronic, recurrent, inflammatory disease located in intertriginous areas, and significantly impairs patients' quality of life. The disease has a prevalence estimated of about $1 \%$. The grading of HS is mainly based on clinical assessment (1). Some scales and scoring systems exist for the evaluation of the disease severity and treatment response, however they have limitations $(1,2)$. There is a need for identifying novel biomarkers, which could be used in daily practice to assess

This article is freely accessible online.

Correspondence to: Prof. Jacek C. Szepetowski, Department of Dermatology, Venereology and Allergology, Wrocław Medical University, ul. Chałubińskiego1, 50-368 Wrocław, Poland. Tel: +48 717842286, e-mail: jacek.szepietowski@umed.wroc.pl

Key Words: Chitinase-3-like protein 1, YKL-40, hidradenitis suppurativa, biomarker, tissue expression, inflammation. disease stage, helping with choosing the treatment options and estimating the progression of the disease. Recently, we suggested a new biomarker, chitinase-3-like protein 1 (YKL40), which serum level was elevated in patients with HS and correlated with disease activity (3). YKL-40 is a member of a conservative family of mammalian chitinases, however it lost the enzymatic activity (4). Its role is not fully understood. Nevertheless, this protein is considered to play a role in inflammation, proliferation and carcinogenesis. It is expressed and secreted by activated neutrophils, monocytes and macrophages (5). YKL-40 was evaluated in many inflammatory and malignant conditions, like rheumatoid arthritis, inflammatory bowel disease, cardiovascular diseases and many cancers $(6,7)$. It was also examined in some dermatological diseases $(8,9)$. Since we demonstrated elevated serum levels of YKL-40 in HS, we aimed to explore the expression of this protein in the diseased tissue. Thus, this pilot study, was undertaken in continuation of our previous research.

\section{Materials and Methods}

The study was conducted on a group of 7 patients suffering from HS diagnosed according to clinical criteria. All patients enrolled to the study were eligible and included in the final analysis. Each patient underwent full physical examination. Then biopsies were taken from lesional skin and non-lesional surrounding skin. The group consisted of three women and four men, aged from 18 to 68 years (mean $37.1 \pm 17.05$ years). The body mass index ranged from 21.71 to $37.65 \mathrm{~kg} / \mathrm{m}^{2}$ (mean $28.6 \pm 6.6 \mathrm{~kg} / \mathrm{m}^{2}$ ). Five patients $(71.1 \%$ ) were qualified as overweight or obese. The disease duration ranged from 3 to 10 years. Four patients $(57.1 \%)$ were active or exsmokers. The severity of the disease was assessed according to Hurley grading system - four patients had stage II and three patients had stage III disease.

The tissue expression of YKL-40 was assessed using an immunohistochemical method. Immunohistochemical reactions (IHC) were performed on $4 \mu \mathrm{m}$-thick formalin-fixed paraffinembedded tissue sections on the detection platform Dako AutostainerLink 48. The slides were deparaffinized, rehydrated and subjected to target retrieval in the pretreatment module, 

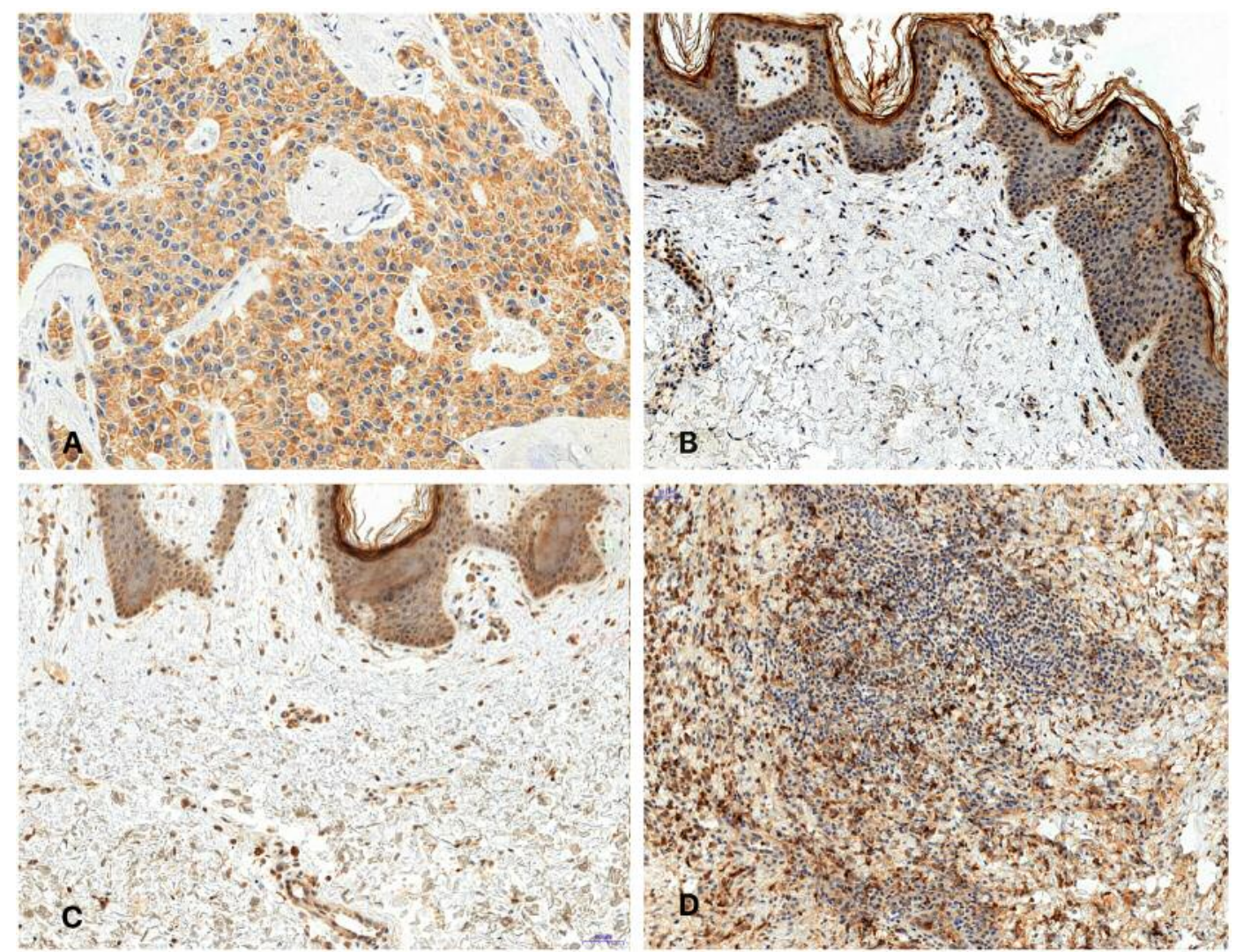

Figure 1. Expression of YKL-40. (A) Strong positive IHC reaction (cytoplasmic brown colored staining) for YKL-40 in breast cancer cells. (B) Physiological YKL-40 expression in healthy skin. (C) YKL-40 expression in non-lesioned skin. (D) Positive IHC reaction in inflammatory cells of the infiltrates in lesioned skin in hidradenitis suppurativa. Magnification is $\times 20$.

PTLink (Dako, Glostrup, Denmark) at $95^{\circ} \mathrm{C}$ in Target Retrieval Solution High pH (Dako). Then the slides were cooled in TBS with $0.1 \%$ Tween (TBS-T). IHC reactions were performed using ImmPRESS Reagent Kit, Peroxidase, Anti-Goat IgG (Vector Laboratories, Burlingame, CA, USA). During the first stage, the endogenous peroxidase activity was quenched by incubation in Peroxidase-Blocking Reagent for $5 \mathrm{~min}$. After rinsing in TBS-T, the slides were incubated with Normal Horse Serum for 20 min and subsequently with anti-Chitinase-3-like Protein 1 immunoglobulin (R\&D Systems, catalog no. AF2599) diluted 1:50 in Antibody Diluent (for $30 \mathrm{~min}$ at room temperature). The slides were rinsed in TBS-T and incubated with secondary antibodies for $30 \mathrm{~min}$. After rinsing in TBS-T, the slides were incubated for $10 \mathrm{~min}$ with diaminobenzidine. The slides were rinsed once again in TBS-T. Then the slides were counterstained with hematoxylin, rinsed in distilled water and dehydrated in graded ethanol alcohols and xylene. The expression of YKL-40 was evaluated under the light microscope at 10 times and 20 times magnification.

The invasive ductal breast cancer tissue (two biopsies), wellknown to express YKL-40, served as a positive control. Moreover, healthy skin tissue (two biopsies) was used as an additional control. There was no statistical analysis performed due to relatively small number of samples.
The study was conducted in compliance with ethic regulations and follows the principles of the Declaration of Helsinki. The study has been approved by the Bioethics Committee of the Wrocław Medical University (opinion no. 153/2017).

\section{Results}

The breast cancer cells showed a strong expression of YKL40. A positive cytoplasmic reaction was seen within the whole solid tissue of the cancer. Healthy control skin presented just the epidermal YKL-40 expression mainly within the basal layer (Figure 1A and B).

In non-lesional HS skin, the pattern of YKL-40 expression was similar to that observed in healthy skin. There were no or few inflammatory cells showing positive IHC reaction (Figure 1C).

Inflammatory infiltrates of various grades were visible in all samples of lesioned skin of HS. The inflammatory cells within those infiltrates, mainly leukocytes and lymphocytes, showed a strong cytoplasmic expression of YKL-40, which was observed in all patients (Figure 1D). 


\section{Discussion}

The present paper is a continuation of our recent research on the role of YKL-40 in HS and its usefulness as a biomarker of disease severity. In our previous study, YKL-40 was found significantly elevated in the serum of patients with HS (3). Moreover, the serum levels of this protein reflected the severity of the disease assessed with the Hurley grading system. There were significant differences between YKL-40 serum levels in HS patients classified as Hurley I and III, or Hurley II and III. The levels of YKL-40 correlated also with biomarkers of inflammation like $\mathrm{C}$-reactive protein (CRP) and white blood cells (WBC). However, YKL-40 seemed to be a better marker for monitoring the grade of inflammation in HS, because CRP and WBC stayed within normal ranges in large proportion of our patients. We have also discussed whether the elevated YKL-40 serum levels in HS was the result of proinflammatory cytokines involvement and migration of phagocytic cells to the lesioned skin with the development of local inflammation and coexisting bacterial superinfection or was associated with a systemic inflammatory response (10).

In the current study, the inflammatory cells within the lesioned skin of HS presented a strong expression of YKL40. These findings confirm that the local inflammatory infiltrates could be an important source of YKL-40, leading to higher serum levels of this protein in patients with HS.

Other molecules, that have been proposed as biomarkers of HS, are soluble IL-2 receptor (sIL-2R), myeloid marker S100A8/A9 and cytokines like IL-6, IL-17 or IL-23 (11-14). However, the correlation between those biomarkers and severity of the disease is not confirmed.

Our hypothesis is that skin lesions in HS contribute totally in or at least are a significant part of elevated YKL-40 serum levels. In light of our results, YKL-40 can be considered as one of the most valuable biomarkers, so far known, for the assessment of HS severity and degree of inflammation in this disease.

\section{References}

1 Jemec GB: Biomarkers in hidradenitis suppurativa. Br J Dermatol 168: 1151-1153, 2013.

2 Kimball AB, Jemec GB, Yang M, Kageleiry A, Signorovitch JE, Okun MM, Gu Y, Wang K, Mulani $\mathrm{P}$ and Sundaram M: Assessing the validity, responsiveness, and meaningfulness of the Hidradenitis Suppurativa Clinical Response (HiSCR) as the clinical endpoint for hidradenitis suppurativa treatment. Br J Dermatol 171: 1434-1442, 2014.
3 Matusiak Ł, Salomon J, Nowicka-Suszko D, Bieniek A and Szepietowski JC: Chitinase-3-like protein 1 (YKL-40): novel biomarker of hidradenitis suppurativa disease activity? Acta Derm Venereol 95: 736-737, 2015.

4 Aronson NN, Blanchard CJ and Madura JD: Homology modeling of glycosyl hydrolase family 18 enzymes and proteins. J Chem Inf Comput Sci 37: 999-1005, 1997.

5 Hashimoto S, Suzuki T, Dong HY, Yamazaki N and Matsushima $\mathrm{K}$ : Serial analysis of gene expression in human monocytes and macrophages. Blood 94: 837-844, 1999.

6 Johansen JS, Jensen BV, Roslind A, Nielsen D and Price PA: Serum YKL-40, a new prognostic biomarker in cancer patients? Cancer Epidemiol Biomarkers Prev 15: 194-202, 2006.

7 Prakash M, Bodas M, Prakash D, Nawani N, Khetmalas M, Mandal A and Eriksson C: Diverse pathological implications of YKL-40: answers may lie in 'outside-in' signaling. Cell Signal 25: 1567-1573, 2013.

8 Salomon J, Matusiak $€$, Nowicka-Suszko D and Szepietowski JC: Chitinase-3-Like Protein 1 (YKL-40) Reflects the Severity of Symptoms in Atopic Dermatitis. J Immunol Res 2017: 5746031, 2017.

9 Salomon J, Matusiak $\succeq$, Nowicka-Suszko D and Szepietowski JC: Chitinase-3-Like Protein 1 (YKL-40) Is a New Biomarker of Inflammation in Psoriasis. Mediators Inflamm 2017: 9538451, 2017.

10 van der Zee HH, de Ruiter L, van der Broecke DG, Dik WA, Laman JD and Prens EP: Elevated levels of tumour necrosis factor (TNF)- $\alpha$, interleukin (IL)- $1 \beta$ and IL-10 in hidradenitis suppurativa skin: a rationale for targeting TNF- $\alpha$ and IL- $1 \beta$. Br J Dermatol 164: 1292-1298, 2011.

11 Matusiak $€$, Bieniek A and Szepietowski JC: Soluble interleukin-2 receptor serum level is a useful marker of hidradenitis suppurativa clinical staging. Biomarkers 14: 432437, 2009.

12 Wieland CW, Vogl T, Ordelman A, Vloedgraven HG, Verwoolde LH, Rensen JM, Roth J, Boer J and Hessels J: Myeloid marker S100A 8/A 9 and lymphocyte marker, soluble interleukin 2 receptor: biomarkers of hidradenitis suppurativa disease activity? Br J Dermatol 168: 1252-1258, 2013.

13 Jiménez-Gallo D, de la Varga-Martínez R, Ossorio-García L, Albarrán-Planelles C, Rodríguez C and Linares-Barrios M: The clinical significance of increased serum proinflammatory cytokines, C-reactive protein, and erythrocyte sedimentation rate in patients with hidradenitis suppurativa. Mediators Inflamm 2017: 2450401, 2017.

14 Matusiak Ł, Szczęch J, Bieniek A, Nowicka-Suszko D and Szepietowski JC: Increased interleukin (IL)-17 serum levels in patients with hidradenitis suppurativa: Implications for treatment with anti-IL-17 agents. J Am Acad Dermatol 76: 670-675, 2017.

Received September 24, 2018

Revised October 12, 2018

Accepted October 15, 2018 